\title{
Causation or confounding: why controls are critical for characterizing long COVID
}

To the Editor-Early reports of persisting symptoms following COVID-19 signaled a debilitating long-term fate for an undetermined proportion of people who survive COVID-19 ${ }^{1}$. A year later, there is no consensus on a name, a duration or symptoms for this syndrome, known alternatively as 'post-acute COVID-19', 'post-COVID syndrome' or 'long COVID'. At the time of this writing, between 170 and 205 persistent, intermittent and relapsing symptoms have been attributed to long COVID, lasting anywhere between 4 weeks and $>1$ year after infection with the coronavirus SARS-CoV-2 ${ }^{2}$. Many of the self-reported symptoms, such as intermittent headaches or tiredness, are nonspecific and prevalent in the general population, whereas others, such as an inability to yawn or chapped lips, are biologically implausible, and are unlikely to meet Bradford Hill criteria for causality ${ }^{3}$.

The finding that some patients develop post-infectious complications or neuropsychological sequelae as a consequence of infection and inflammation of body organ systems, through as-yet poorly understood pathophysiological mechanisms, is not unexpected and has been reported after other infectious diseases, particularly glandular fever and influenza. It is, however, the projected burden that is unprecedented. In the UK, the Office for National Statistics estimated that 1.1 million people (1.7\% of the UK population) had long COVID in March 2021, on the basis of the number of people self-reporting long COVID, defined as "experiencing symptoms $>4$ weeks" after COVID-19" 4 .

Studies of long COVID thus far-nearly all from high-income countries-have been difficult to interpret or compare (Fig. 1), not only because of the lack of a consistent case definition but also because of systematic selection bias of cases, often without appropriate controls ${ }^{5}$. Unsurprisingly, hospitalized patients with COVID-19, especially those who require prolonged intensive care, have reported very high rates of persistent symptoms, even though the vast majority of the more than 136 million cases worldwide occurred in healthy working-age, non-hospitalized adults, with mild to moderate self-limited illness. Meanwhile, community-based studies have raised questions about the representativeness of cases compared with
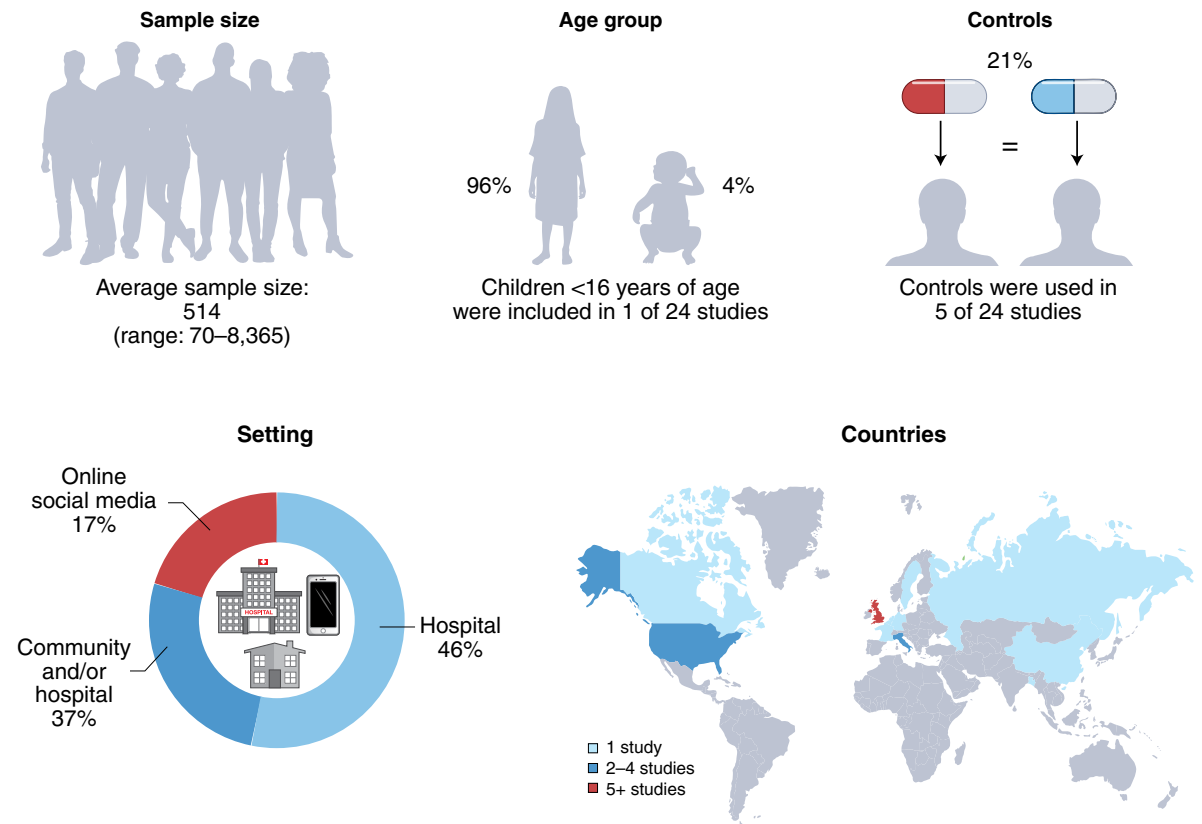

Fig. 1 | Visual summary of characteristics of studies on long COVID up to 15 April 2021. The studies varied in sample size, age range, use of controls, and the settings and countries represented.

the general population, especially those using online recruitment, with many studies relying on self-reported symptoms and without laboratory-confirmed diagnosis of SARS-CoV-2 infection. Selection bias becomes even more pronounced when people are recruited through online support groups, where very high rates of often nonspecific long-term symptoms have been reported, even in children ${ }^{6}$. These biases are further compounded in systematic reviews of published studies, with estimates of up to $80 \%$ (95\% confidence interval, 65-92\%) of patients with COVID-19 reported to have one or more long-term symptoms after acute infection?

Another major limitation is the lack of appropriate controls with which to objectively identify and quantify attributable symptoms, especially in assessment of longer-term sequelae. Some studies have included symptomatic but SARS-CoV-2 test-negative people as control participants, whereas others have compared patients with persistent symptoms with those with short-lasting illness (Table 1). Longer-term assessment, however, requires additional controls for assessment of the added burden of long COVID symptoms in patients with
COVID-19 compared with the general population, not only because many of the reported symptoms of long COVID are nonspecific and prevalent in the general population $^{8}$, but also because some reported symptoms have been intermittent or relapsing or have emerged several weeks to months after infection.

To address some of these biases, we $\mathrm{e}^{9}$ and others ${ }^{10}$ used established longitudinal cohorts recruited at the start of the pandemic for regular testing for antibodies to SARS-CoV-2 and thus provided objective confirmation of infection. We asked our healthcare-worker participants about 72 symptoms reported to be associated with long COVID ${ }^{9}$, at a median of 7.5 months after the development of COVID-19, and found that mental-health, gastrointestinal and dermatological symptoms were as common among 140 patients with mild-to-moderate symptomatic seropositive COVID-19 ('cases') as in 1,160 control participants who remained asymptomatic and seronegative throughout the surveillance period ${ }^{9}$. Of concern, $40-60 \%$ of both cases and control participants reported mental-health symptoms, which highlights the toll of pandemic on the healthcare 
Table 1 | Characteristics of studies on long COVID up to 15 April 2021

\begin{tabular}{|c|c|c|}
\hline \multicolumn{2}{|l|}{ Characteristics } & \multirow{2}{*}{$\begin{array}{l}\text { Number of studies (\%) } \\
3(13 \%)\end{array}$} \\
\hline Location & International and/or multiple & \\
\hline & Europe & $14(58 \%)$ \\
\hline & United States & $4(17 \%)$ \\
\hline & Indian sub-continent & $2(8 \%)$ \\
\hline & Southeast Asia & $1(4 \%)$ \\
\hline \multirow[t]{5}{*}{ Sample size } & $\geq 3,000$ & $3(13 \%)$ \\
\hline & $2,000-2,999$ & $3(13 \%)$ \\
\hline & $1,000-1,999$ & $3(13 \%)$ \\
\hline & $500-999$ & $1(4 \%)$ \\
\hline & $<500$ & $14(58 \%)$ \\
\hline \multirow[t]{4}{*}{ Setting } & Multi-center, hospital & $3(13 \%)$ \\
\hline & Single-center, hospital & $8(33 \%)$ \\
\hline & Community and/or hospital & $9(38 \%)$ \\
\hline & Online and/or social media & $4(17 \%)$ \\
\hline \multirow[t]{2}{*}{ Age group } & Adults & $23(96 \%)$ \\
\hline & Children & $1(4 \%)$ \\
\hline Female \% (mean) & $53 \%$ & \\
\hline \multirow{6}{*}{$\begin{array}{l}\text { Most common } \\
\text { symptom }\end{array}$} & Fatigue & $19(79 \%)$ \\
\hline & Anosmia & $1(4 \%)$ \\
\hline & Diarrhea & $1(4 \%)$ \\
\hline & Dyspnea & $1(4 \%)$ \\
\hline & Insomnia & $1(4 \%)$ \\
\hline & Muscle ache & $1(4 \%)$ \\
\hline \multirow[t]{4}{*}{ Study design } & Longitudinal & $4(17 \%)$ \\
\hline & Retrospective cohort & $10(42 \%)$ \\
\hline & Prospective cohort & $2(8 \%)$ \\
\hline & Cross-sectional & $8(33 \%)$ \\
\hline \multirow[t]{2}{*}{ Testing method } & Only laboratory confirmed (RT-PCR and/or antibody) & $16(67 \%)$ \\
\hline & $\begin{array}{l}\text { Clinical diagnosis, suspected or laboratory-confirmed } \\
\text { (RT-PCR and/or antibody) COVID-19 }\end{array}$ & $8(33 \%)$ \\
\hline \multirow[t]{4}{*}{ Start of follow-up } & After hospital discharge & $6(25 \%)$ \\
\hline & After onset of symptoms & $13(54 \%)$ \\
\hline & After RT-PCR and/or antibody test & $4(17 \%)$ \\
\hline & Not reported & $1(4 \%)$ \\
\hline \multirow[t]{2}{*}{ Control group } & Non-matched control participants & $2(8 \%)$ \\
\hline & Matched control participants & $3(13 \%)$ \\
\hline \multirow{3}{*}{$\begin{array}{l}\text { Follow-up } \\
\text { maximum interval }\end{array}$} & $<6$ months & $14(58 \%)$ \\
\hline & $\geq 6$ months & $7(29 \%)$ \\
\hline & Not reported & $3(13 \%)$ \\
\hline
\end{tabular}

Studies ( $n=24$ ) on long COVID up to 15 April 2021, found through systematic searches on PubMED and MedRxiv with key terms 'long COVID' or 'post COVID' or 'persisting symptom'. Five studies were in pre-print and had not undergone peer review. Percentages have been rounded and therefore may not total to $100 \%$

workforce. We also identified three clusters that included 12 symptoms-affecting the sensory, neurological and cardiorespiratory systems-that were reported by $67 \%$ of cases but also $44 \%$ of control participants, which emphasizes the ongoing difficulties in characterizing long COVID. The risk difference of $23 \%$ more than 6 months after infection, however, demonstrates the substantial long-term burden of COVID-19 in predominantly healthy adults. A similar Swedish study also estimated a higher prevalence of symptoms after 8 months in seropositive healthcare workers with mild COVID-19 than in seronegative healthcare workers, with seropositive cases reporting considerable disruption to their work, social and home life ${ }^{10}$.

It is increasingly apparent that the long-term impact of this pandemic will be considerable and will substantially affect quality of life. Current best-guess estimates for long COVID (defined as self-reported persistence of any symptoms for 12 weeks or more after COVID-19) in population-based UK studies range from $2.2 \%$ (ref. ${ }^{8}$ ) to $13.7 \%$ (ref. ${ }^{4}$ ), and systematic assessment of available data identify female sex, middle age, white ethnicity and specific underlying co-morbidities as potential risk factors ${ }^{7}$. More robust, well-conducted longitudinal studies are urgently needed for proper characterization of this syndrome to inform clinicians, to develop evidence-based guidelines and to direct healthcare resources to where they are most needed.

\section{Zahin Amin-Chowdhury ${ }^{1}$ and Shamez N. Ladhani $i^{1,2} \bowtie$ \\ ${ }^{1}$ Immunisation and Countermeasures Division, Public Health England, London, UK. ${ }^{2} P a e d i a t r i c$ Infectious Diseases Research Group, St. George's University of London, London, UK.

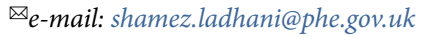

Published online: 17 June 2021

https://doi.org/10.1038/s41591-021-01402-w

\section{References}

1. Carfl, A., Bernabei, R. \& Landi, F. J. Am. Med. Assoc. 324, 603-605 (2020)

2. Davis, H.E., et al. Preprint at medRxiv https://doi.org/ 10.1101/2020.12.24.20248802 (5 April 2021).

3. Li, K. et al. Reprod. Biomed. Online 42, 260-267 (2021).

4. Office for National Statistics. https://www.ons.gov.uk/ peoplepopulationandcommunity/healthandsocialcare/ conditionsanddiseases/bulletins/prevalenceofongoingsymptoms followingcoronaviruscovid19infectionintheuk/1april2021 (accessed 14 April 2021).

Rando, H.M., et al. Preprint at medRxiv https://doi. org/10.1101/2021.03.20.21253896 (2021).

6. Buonsenso, D., Pujol, F. E., Munblit, D., Mcfarland, S. \& Simpson, F. Preprint at https://doi.org/10.20944/ preprints202103.0271.v1 (2021).

7. Lopez-Leon, S. et al. Preprint at medRxiv https://doi.org/ 10.1101/2021.01.27.21250617 (2021).

Sudre, C. H. et al. Nat. Med. 27, 626-631 (2021).

9. Amin-Chowdhury, Z., et al. Preprint at medRxiv https://doi.org/ 10.1101/2021.03.18.21253633 (16 April 2021).

10. Havervall, S. et al. J. Am. Med. Assoc. 325, 2015-2016 (2021).

Competing interests

The authors declare no competing interests. 